\title{
Factores Curativos en psicoterapia de grupo: su evaluación y análisis en una muestra de alcohólicos rehabilitados*
}

\author{
Colli Alonso,M. 1; Zaldívar Pérez,D.2
}

(1) Psicólogo, M. Sc., y Profesor del Departamento de Psicología-Sección Adicciones, Departamento de Tratamientos Especializados Ambulatorios, Hospital Psiquiátrico de La Habana, Cuba. email: mcolli@infomed.sld.cu

(2) Dr. Sc., Profesor, y Decano de la Facultad de Psicología, Universidad de La Habana. Centro de Atención y Atención a la Población

M. Sc. Ps. Mario Colli Alonso, Departamento de Tratamientos Especializados, Ave. Boyeros y Ave 26, Calzada del Cerro, Hospital Psiquiátrico de La Habana, Cuba. email: mcolli@infomed.sld.cu

\section{RESUMEN}

Se parte de la idea de los efectos positivos de la terapia de grupo de alcohólicos en la literatura y se plantea la evaluación de los resultados de la psicoterapia de grupo en una muestra de pacientes alcohólicos $(n=34)$, tratados ambulatoriamente con un tiempo de abstinencia entre los tres meses y los tres años y medio, con el método del Cuestionario de Factores Curativos de Yalom (60 ítems), encontrándose los Factores Curativos de grupo 1) Altruismo, 2) Comprensión de sí mismo, 3) Revalidación de la Familia, según la teoría de Yalom, entre los de más alto puntaje, comparándose estos resultados con los reportados anteriormente en la literatura del tema del grupo de Yalom, y también con un grupo control de pacientes psiquiátri$\cos (n=19)$, estableciéndose las correspondientes diferencias. Se establecen también inferencias útiles para la psicoterapia de grupo de pacientes alcohólicos y las necesidades de este en la terapia.

Palabras clave: Psicoterapia de grupo, Tratamiento alcoholismo, evaluación, Cuestionario de Yalom.

\section{ABSTRACT}

Taking the idea of the positive results of alcoholic group therapy found in the literature as our starting point, we undertook an evaluation of the results of group psychotherapy in a sample of alcoholic patients $(n=34)$, treated as outpatients, with an abstinence time of three months to three and a half years, utilising the Yalom Curative Factors Questionnaire 160 items). We found the Group Curative Factors to be: 1) Altruism, 1) Understanding of oneself, 3) Revalidation of the family, according to Yalom's Theory, among those with the highest score. These results were compared with those reported previously in the literature on the subject of Yalom's group therapy, and also with a control group of psychiatric patients $(n=19)$, establishing the corresponding differences. Useful inferences are also established for group psychotherapy for alcoholic patients and their needs during therapy.

Key words: Group psychotherapy; Alcoholism treatment, Evaluation, control group, Yalom's, Questionnaire.

\section{INTRODUCCIÓN}

\section{Antecedentes de la investigación.}

L a revisión de la información existente en psicoterapia de grupo nos lleva a nuestro trabajo, para el cual primero expondremos los antecedentes que tiene con respecto a la psicoterapia de grupo en general, y después los antecedentes que tiene con relación a la terapia de grupo en alcohólicos y otros adictos en la literatura del tema, el cual tiene las siguientes razones:
1) La psicoterapia de grupo es evaluable, lo cual se recoge en Pattison (1965) sobre el tema, autor que hace un artículo antológico sobre la dificultad para evaluar esta, examinando la literatura hasta esa fecha del tema y recogiendo tres criterios para evaluarla: 1) Psicométrico, 2) Conductual y 3) De Constructo, a lo que nosotros hemos agregado que es el de 4) Procesos de grupo (Colli, Zaldívar, 2002) y el que se recoge en otros autores (Bach, 1958; Potts, 1960; Yalom, 1986; McKenzie, 1990) y al que actual-

* Versión Actualizada de Tesis de Máster en Psicología Clínica, Univ. de La Habana(2000).Primera Versión presentada en el Congreso de Psicología, Ciencias y Tecnologías de la Salud en Ciudad Guatemala, Mayo/2000, versión actual presentada en el Simposio Internacional de Adicciones, PSICO- 2000, La Habana, 2000. 
mente pertenecen una gran cantidad de trabajos en Estados Unidos y Canadá, y casi ninguno en países hispanos -excepto dos en España-, basándose unos en el grupo pequeño (small group) y otros en los Factores Curativos. En los trabajos realizados en Cuba sobre el tema de la evaluación Grupal, debe señalarse el de Rodríguez (1997) que planteó pruebas psicométricas para ello, o sea un enfoque psicométrico para ello, pero esto no se relaciona con nuestro interés de enfoque, que es el de los procesos de grupo. Más recientemente existe en la literatura la combinación entre el enfoque psicométrico y el enfoque de procesos de grupo, es decir una integración de enfoques para evaluar los grupos, (Joyce, Azim, Morin, 1988; Smith, McNiel, Binder, 1988; Tschuschke, Dies, 1997; McKenzie, Tschuschke, 1993; Tschuschke, Dies, 1994), según ha sido recogido y analizado por nosotros, el cual nos parece el más objetivo en la actualidad para evaluar resultados de grupo.

2) Desde el punto de vista del paciente, en Bach (1952;1958), en Potts (1960), existe un enfoque sociométrico, mientras que en Yalom (1986) y McKenzie (1990), existe la propuesta de un instrumento para evaluar el grupo desde el paciente, pero desde los Factores Curativos producidos por el grupo y percibidos por el paciente, partiendo de que sí queremos saber qué ocurre desde el punto de vista del paciente en el grupo había que buscar el instrumento que satisficiera este objeto.

3) En la literatura nacional e internacional sobre terapia de grupo de alcohólicos, no existía un instrumento que propusiera la evaluación de los efectos del grupo sobre el paciente alcohólico, excepto el Cuestionario de Yalom según había recogido Flores (1997).

4) Se ha escrito sobre técnicas de grupo para alcohólicos, pero no de la evaluación en esta área. Se ha revisado a autores de distintas latitudes (ver referencias bibliográficas), pero no encontramos técnicas de evaluación de grupo.

5) La única solución posible era aplicar el instrumento más adecuado que se encontrara, y validarlo para alcohólicos.

Así la consulta de dichos materiales y de algunas fuentes vivas, ha permitido orientarnos hacia el área de la investigación en psicoterapia de grupo, donde en el área internacional se lleva la palma el grupo de Yalom (1986), el cual ha orientado su interés investigativo a la presencia de los mecanismos de grupo o Factores Curativos de grupo, como productores del cambio psicológico/psicoterapéutico de los pacientes de grupo, simultáneamente proponiendo un instrumento para la evaluación de este cambio, el cual se conoce con el nombre de Cuestionario de Factores Curativos de grupo, el que comenzó a utilizarse en contexto clínico a partir de 1975, y del que aún hoy hemos encontrado evidencia de su uso en varias áreas de la psicoterapia de grupo. Este instrumento fue creado a los efectos de estudiar los mecanismos o Factores Curativos que producen los grupos, que pueden mejorar a los pacientes que a ellos asisten. Este trabajo tiene por tanto como base la psicoterapia de grupo de orientación interactiva, según Yalom, y es una aplicación de su instrumento. Ahora bien como la psicoterapia de grupo para alcohólicos ha sido defendida por unos y atacada por otros, interesa exponer la evidencia existente a su favor.

\section{Terapia de grupo en Alcoholismo y abuso de sus- tancias. Antecedentes en la literatura.}

Ahora bien por la importancia que para nuestro trabajo tiene la terapia de grupo en pacientes alcohólicos, consideramos necesario hacer un resumen que exponga los antecedentes y actualidad de esta, así como las investigaciones que avalan sus resultados positivos.

Reporta Vannicelli (1995), que sobre las últimas 4 décadas la psicoterapia de grupo ha ido ganando terreno e incrementándose su aceptación en la modalidad de consumidores de sustancias, que si aplicamos la terminología de nuestro medio sería toxicómanos (Glez. Menéndez, 1997), y también en la modalidad de tratamiento a sus familiares, recogiendo además que la descripción de técnicas de grupo para estos apareció en la literatura por vez primera en 1946 en McCarthy (Vannicelli, 1995), para miembros de familia de consumidores en 1959 por Igershimer (Vannicelli, 1995), y para adultos hijos de alcohólicos en 1980 por Dietler (Vannicelli, 1995). Existe dentro de la vasta literatura de terapia de grupo de alcohólicos contribuciones en este campo de consumidores colaterales o codependientes (Vannicelli, 1987; 1995) y adultos hijos de alcohólicos (ACOAS) (Brown, 1988; Vannicelli, 1995), así como también existe una vasta literatura que defiende la terapia de grupo en alcohólicos exactamente, ya sea en forma de grupo de discusión, de orientación psicodinámica, cognitivo-conductual, o interaccional (Mullan, 1963; Boga ni, 1984; Sierra, 1978; Mathiasen, Davenport, 1988; Vannicelli, 1982; 1987; 1995; Golden, Halliday, Khantzian, Mc Auliffe, 1995; Khantzian, 1989; Hernández, 1989;Zaldívar, 1998; Monrás et all, 2000; Martínez y Fdez., 1996; Cuadrado, 1998), a lo cual se suman todos aquellos estudios que han combinado la terapia de grupo con otros métodos en alcohólicos y demostrado su eficacia (Alfano, Thurstin, 1989; Glez. Menéndez et all, 1995), por las técnicas empleadas en los mismos, incluso algunos estudios comparten la idea de la com- 
binación con los tratamientos institucionales y manejo de las teorías de los Alcohólicos Anónimos (Brown, 1985; Alfano, Thurstin, 1989), inclusive existiendo estudios que también reportan a los grupos de doble A. A. como efectivos para ciertos tipos de personas, y los alcohólicos que fueron atendidos en estos grupos se reportaron con promedios bajísimos de patologías.

\section{Características de nuestros grupos:}

1) Nuestros grupos son grupos abiertos, pues la patología del Alcoholismo es una enfermedad incurable, que es propensa a recaídas (Marlatt y Gordon, 1985), y entendemos el grupo debe ser abierto.

2) Son grupos grandes, lo cual expresa que se han efectuado con 30-40 personas en cada sesión. El grupo pequeño reproduce la familia y el grande a la sociedad, como ha enfatizado la literatura del grupo grande (Lawrence, 1993; Klein, 1993; Agazarian, Carter, 1993; Carr, 1993; Rodríguez, 1982; 1988; 1997).

3) Son grupos de psicoterapia para la rehabilitación de alcohólicos a largo término (Vannicelli, 1982; 1995).

4) El tipo de grupo terapéutico es esencialmente el de grupos de discusión (Bogani, 1978; Avila Espada, 1993), ya que tiene como objeto centro el estímulo del aprendizaje a través de la discusión de los temas referidos y básicos de Alcoholismo.

5) Nuestra técnica es diversa, tiene elementos de Análisis Transaccional (Berne, 1992; Steiner, 1992; Steiner, 1972), de grupos didácticos, y de A. T. Gestáltico, también de técnicas interactivas siguiendo el modelo de Yalom y discípulos, (Yalom, 1986; Vinogradov y Yalom, 1990; Vannicelli, 1982; 1995, Flores, 1997).

\section{Material y Método}

Se realizó un estudio de tipo exploratorio para conocer en nuestro medio cómo operan los Factores Curativos, en un universo que estaba comprendido por los pacientes alcohólicos que recibían terapia de grupo de carácter ambulatorio en nuestro centro, Departamento de Tratamientos Especializados del Hospital Psiquiátrico de La Habana (sito en Calle Boyeros y Avenida 26, Ciudad Habana).

La muestra está formada por 34 pacientes alcohólicos que asistían regularmente una vez por semana por dos horas, a la terapia de grupo ambulatoria entre los tres meses de abstinencia, como mínimo, y los tres años y medio como máximo. Nos interesaban qué Factores Curativos grupales, han resultado relevantes a la hora de determinar el fenómeno de la abstinencia alcohólica, interesante, y difícil para que un alcohólico deje de beber, o lo que es lo mismo logre la abstinencia. El eje del trabajo descansa sobre la hipó- tesis de que los Factores Curativos que produce el propio grupo hacen cambiar al paciente y producen la abstinencia. Se utilizó también un grupo control de 19 pacientes psiquiátricos: 6 Trastornos de Personalidad, 3 esquizoides, 2 Paranoides, 1 Obsesivo-compulsivo, 10 con Desórdenes Psiconeuróticos, y 3 con Trastorno Situacional Transitorio de Ansiedad, que habían recibido psicoterapia de grupo por tres meses, una vez por semana, en el Departamento de Psiquiatría, del Hospital "Joaquín Albarrán", ubicado en Ave 26 entre Ave Boyeros y Calzada del Cerro.

Criterios de inclusión:

1) Pacientes alcohólicos diagnosticados por la CIE10, OMS. (Martín, 1992), según los indicadores siguientes:

F-10: Trastornos mentales y del comportamiento debidos al consumo de alcohol.

F-10.2: Síndrome de Dependencia.

2) Pacientes alcohólicos sometidos a terapia de grupo al menos tres meses, y a lo máximo tres años y medio, y con el mismo tiempo de abstinencia que de asistencia a los grupos, ya que es condición requerida estar en abstinencia para entrar al grupo.

3) Edad: Entre los 25 y los 60 años de edad, ya que es el marco de rehabilitación y eclosión más usual en la enfermedad.

4) Sexo: Fueron encuestados de los 34 pacientes, 32 hombres y 2 mujeres, por la razón de que existen mucho menos mujeres alcohólicas que hombres, y también llegan mucho menos las mujeres al tratamiento.

5) Escolaridad: Media.

6) Lugar de residencia: En general se encuestó pacientes de la Ciudad de La Habana, y provincia Habana, dos casos de provincias orientales, y uno de Santa Clara.

Criterios de exclusión:

1) Se excluyeron pacientes alcohólicos, aún en abstinencia que no hubieran recibido terapia de grupo.

2) Se excluyeron retrasados mentales, y con trastornos psicóticos.

\section{Técnicas o instrumentos:}

1) Para dar cumplimiento a los objetivos se utilizó el Cuestionario de Yalom para Factores Curativos de grupo (1986), de 60 ítems, de seis respuestas posibles, que en nuestro caso consideramos en un rango de $12,10,8,6,4$,

2. [Doce equivalía a lo más útil; diez equivalía a extremadamente útil; ocho equivalía a muy útil; seis equivalía a útil; cuatro equivalía a poco útil, y dos equivalía a menos útil], el cual recoge e integra un enfoque ecléctico e interactivo de 
grupo, donde se encuentran resumidos en forma de ítems los mecanismos curativos recogidos en grupos por todo un relevante grupo de terapeutas de grupo, en su práctica clínica en grupos a lo largo de más de 40 años, y por tanto por ser el instrumento que más se adaptaba en la literatura a nuestros objetivos, y funcionaba desde el punto de vista del paciente.

Los ítems de cada factor son los siguientes:

1) Altruismo: $1-5$

2) Cohesión de grupo: 6-10

3) Universalidad: 11-15

4) Aprendizaje interpersonal Insumo: 16-20

5) Aprendizaje Interpersonal Producto: 21-25

6) Guía: 26-30

7) Catarsis: $31-35$

8) Identificación: 36-40

9) Revalidación de la familia: 41-45

10) Comprensión de sí mismo: $46-50$

11) Infundir esperanzas: $51-55$

12) Factores Existenciales: 56-60.

Variables a controlar:

Tipo de tratamiento: Se refiere a si la terapia de grupo ha sido ambulatoria o ingresada, ya que puede ser que influya en que unos Factores Curativos operen y otros no.

Abstinencia total: Es necesario ya que es lo que define que el alcohólico comience su rehabilitación. Se controló observando que todos los pacientes en el tiempo del estudio mantuvieran su abstinencia.

Resultados obtenidos:

Se procesaron los resultados a través del paquete estadístico Statistic, y se utilizó el cálculo de medias (mean), y el estadígrafo T-Student para la obtención de lo siguiente:
En el grupo de Alcohólicos los Factores Curativos más altos en puntuación fueron los siguientes:

1) Altruismo. (F 1).

2) Comprensión de sí mismo (F10)

3) Revalidación de la familia. (F9).

4) Aprendizaje Interpersonal Insumo (F4).

5) Cohesión del grupo (F2).

En el grupo de pacientes psiquiátricos:

1) Altruismo (F1).

2) Comprensión de sí mismo. (F10). Aprendizaje Interpersonal Insumo (F4).

3) Revalidación de la familia (F9).

4) Identificación (F8).

5) Aprendizaje Interpersonal Producto (F5).

Aunque a los efectos de nuestro trabajo sólo nos interesaremos por los cinco factores de más alto puntaje, se presenta una comparación entre las medias obtenidas de los doce Factores Curativos, para su mejor comprensión verlo en Tabla 1.

3) Se recogieron los 10 ítems más importantes para el grupo de alcohólicos, por ser los que nos aportaron información realmente relevante, de los que más adelante se hará un análisis cualitativo:

Los 10 ítems más elevados en Alcohólicos:

1) It 2 "Pertenecer al grupo y ser aceptado por este (Altruismo).

2) It 51 "Saber que no soy diferente a los demás" (Infundir esperanzas).

3) It 21 “Estar en el grupo me ayudó a comprender los problemas del pasado (Aprendizaje Int. Producto).

4) It 22 "Saber por qué pienso y siento como lo hago (Aprendizaje Int. Producto).

Tabla I: Comparación de las medias de los FACTORES CURATIVOS

\begin{tabular}{|lcccc|}
\hline Factores: & $\begin{array}{c}\text { Psiquiátricos } \\
\text { Medias: }\end{array}$ & $\begin{array}{c}\text { Alcohólicos } \\
\text { Medias: }\end{array}$ & T-Student: & P: \\
\hline 1) Altruismo & $8.715789^{*}$ & $9.552941^{*}$ & $-2.39499^{*}$ & $.020332^{*}$ \\
2) Cohesión de grupo & $7.936842^{*}$ & $9.235294^{*}$ & $-2.82416^{*}$ & $.006747^{*}$ \\
3) Universalidad & $7.347368^{*}$ & $8.082353^{*}$ & $-1.44728^{*}$ & $.153938^{*}$ \\
4) Aprendizaje Interpersonal Insumo & $8.610526^{*}$ & $9.247059^{*}$ & $-1.373364^{*}$ & $.175563^{*}$ \\
5) Aprendizaje Interpersonal Producto & $8.294737^{*}$ & $8.882353^{*}$ & $-1.25096^{*}$ & $.216656^{*}$ \\
6) Guía & $7.810526^{*}$ & $8.717647^{*}$ & $-1.67882^{*}$ & $.099304^{*}$ \\
7) Catarsis & $7.326316^{*}$ & $9.0941118^{*}$ & $-3.50075^{*}$ & $.000973^{*}$ \\
8) Identificación & $8.442105^{*}$ & $9.164706^{*}$ & $-1.53718^{*}$ & $.130430^{*}$ \\
9) Revalidación de la Familia & $8.568421^{*}$ & $9.3411176^{*}$ & $-1.58251^{*}$ & $.119715^{*}$ \\
10) Comprensión de sí mismo & $8.610526^{*}$ & $9.352941^{*}$ & $-1.59403^{*}$ & $.117109^{*}$ \\
11) Instalación de esperanza & $8.263158^{*}$ & $8.600000^{*}$ & -.63608 & $.527569^{*}$ \\
12) Factores existenciales & $7.957895^{*}$ & $8.764706^{*}$ & $-1.65932^{*}$ & $.103189^{*}$ \\
\hline * Diferencias Significativas & & & & \\
\hline
\end{tabular}




\section{Figura I: Comparación gráfica de las medias de los Factores Curativos entre psiquiátricos y alcohólicos.}

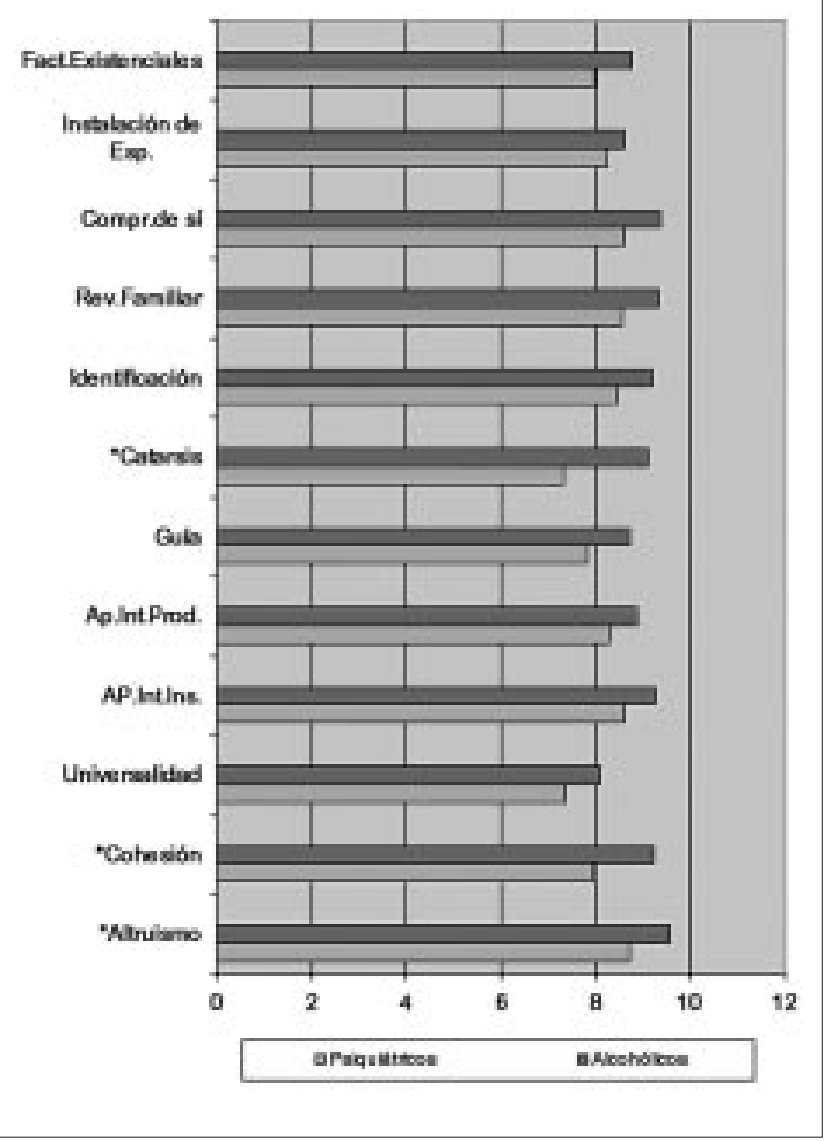

5) It 11 "Advertir que otros mejoraban me alentó" (Universalidad). 55 "Poder decir lo que me molesta en vez de guardar silencio" (Infundir esperanzas).

6) It 38 "Ya no sentirme solo " (Identificación).

7) It 17 "Sentir más confianza en los grupos y otra gente (Aprendizaje Interpersonal Insumo).

8) It 6 "La sugerencia o el consejo del terapeuta para que yo haga algo" (Revalidación de la familia).

9) It 42 "Alguien en el grupo da sus puntos sobre un problema vital " (Rev. de la familia) It 44 "Admirar a mi terapeuta y portarme como él " (Revali. de la familia). It 45 "Estar en mi grupo en cierta forma me ayudó a comprender cómo me crearon en mi familia" (Reval. de la familia).

10) It 10 "Saber que me agradaba o disgustaba una persona por razones que tenían poca relación con mis problemas (Cohesión de grupo). It 50 "Pertenecer a un grupo que me comprendía y me aceptó" (Comprensión de sí mismo)

\section{Discusión y Análisis de los resultados:}

En primer lugar compararemos los datos obtenidos con los obtenidos por otros autores en la literatura del tema.

1) Si comparamos (ver tabla III) estos resultados obtenidos por nosotros con los de Yalom (1986), en su trabajo original, vemos que son muy similares Tabla II.

Tabla II: Comparación entre los resultados clásicos de Yalom en (1986) USA con los obtenidos en Cuba

$\begin{array}{lll}\text { Yalom (1986): } & \text { Alcohólicos: } & \text { Psiquiátricos: } \\ \begin{array}{lll}\text { 1) Altruismo. } & \text { 1) Altruismo. } & \text { 1) Altruismo. } \\ \text { 2) Cohesión grupal. } & \text { 2) Comprensión de sí mismo. } & \text { 2) Comprensión de sí mismo } \\ & \text { Aprendizaje Int. Ins } \\ \text { 3) Universalidad } & \text { 3) Revalidación de la familia. } & \text { 3) Revalidación de la familia. } \\ \text { 4) Aprendizaje Int. ins. } & \text { 4) Aprendizaje Int. Insumo. } & \text { 4) Identificación. } \\ \text { 5) Aprendizaje Int. Prod. } & \text { 5) Cohesión grupal } & \text { 5) Apendizaje Int. Prod. }\end{array}\end{array}$

\section{Comentario sobre la tabla:}

1.1) En nuestro grupo de alcohólicos $(n=34)$, existen 3 grupos de FC recogidos por Yalom (1985), como los más importantes, y en nuestra muestra de psiquiátri$\cos (n=19)$, es curioso que también existen tres grupos entre los cinco que reportó Yalom. El detalle más importante parece ser que en ambas muestras de nuestro estudio el FC más relevante fue el Altruismo, lo cual coincide totalmente con el estudio de Yalom.

1.2) Otro dato interesante es que en nuestras dos muestras coinciden en reportar la cuarteta siguiente: 1) Altruismo ;2) Comprensión de Sí- mismo; 3) Apren- dizaje Int. Insumo; 4) Revalidación de la familia, como dentro de los más altos, diferenciándose únicamente por la presencia del factor curativo Cohesión Grupal, como presente en alcohólicos y ausente en psiquiátricos, y el factor curativo Identificación, presente en psiquiátricos y ausente en alcohólicos. Inclusive los tres primeros lugares de los FC en ambas muestras fueron asignados a los mismos FC de 1) Altruismo, 2) Comprensión de sí mismo ;3) Revalidación de la familia, con la única diferencia de que en la muestra de psiquiátricos es tan importante como Comprensión de sí mismo en segundo lugar el Aprendizaje Int. Insumo. 
1.3) Según McKenzie (1987) los estudios de Yalom las categorías de Factores Curativos: y su grupo han recogido las siguientes frecuencias en

Tabla III: Frecuencias de FACTORES CURATIVOS en la literatura según McKenzie

$\begin{array}{lll}\text { 2) Medianamente usadas: } & \text { 1) Frecuentemente usadas: } & \text { 3) Menos usadas: } \\ \text { 5) Universalidad } & \text { 1) Catarsis } & \text { 9) Comp. de sí mismo. } \\ \text { 6) Altruismo. } & \text { 2) Aprendizaje Int. Ins } & \text { 10) Guía. } \\ \text { 7) Esperanza } & \text { 3) Cohesión grupal. } & \text { 11) Revalidac. de la fam. } \\ \text { 8) Fact. Existenciales. } & \text { 4) Aprendizaje Int. Prod. } & \text { 12) Identificación }\end{array}$

De lo que con respecto a nuestro estudio podemos observar que se recogen en ambas muestras tres de ellos en la muestra de alcohólicos y en la de psiquiátricos están presentes dos de ellos.
1.4) Ahora bien si comparamos nuestro estudio con otro estudio antológico dentro de esta corriente, el de Lescz (1986), con la muestra de este estudio que recibió tto. ambulatorio clasificó los siguientes factores como los más importantes:

\section{Tabla IV: Comparación de los resultados sobre FACTORES CURATIVOS de Lescz en USA y los recogidos en Cuba}

$\begin{array}{lll}\text { Lescz (1986): } & \text { Alcohólicos: } & \text { Psiquiátricos: } \\ \begin{array}{lll}\text { 1) Aprendizaje Inter personal. } & \text { 1) Altruismo } & \text { 1) Altruismo. } \\ \text { 2) Autocomprensión } & \text { 2) Comp. de sí mismo } & \text { 2) Comprensión de s. mismo. } \\ \text { (Comprensión de sí m.) } & & \text { Aprendizaje Int. Ins. } \\ \text { 3) Expresión de sent. (Catarsis) } & \text { 3) Reval. de la fam. } & \text { 3) Reval. de la fam. } \\ \text { 4) Aprendizaje vic. } & \text { 4) Aprendizaje Int. Ins. (Identificación). } & \text { 4) Identificación. } \\ \text { 5) Universalidad. } & \text { 5) Cohesión grupal } & \text { 5) Aprendizaje Int. Prod. }\end{array}\end{array}$

Vemos que se repiten Aprendizaje Interpersonal, Comprensión de sí mismo, en los alcohólicos, y Comprensión de sí mismo, Aprendizaje Int. Ins., Aprendizaje Int. Prod., e Identificación, en los psiquiátricos, lo cual está planteando cambios parecidos en condiciones parecidas. Todavía el grupo de pacientes psiquiátricos tiene más similitud con el de Lescz (1986), lo cual es muy probable que sea porque la muestra es similar, y las diferencias con la muestra de alcohólicos porque la muestra es más diferente.

1.5) Otro dato importante es que McKenzie estableció otras categorías tras revisar exhaustivamente la literatura del grupo de Yalom (1987), tras lo cual estableció la reagrupación siguiente:

Tabla V: Revisión de MacKenzie sobre Categorías de FACTORES CURATIVOS

\begin{tabular}{|c|c|c|}
\hline 1) Trabajo psicológico: & 2) Autorevelación: & 3) Categorías morales no específicas: \\
\hline $\begin{array}{l}\text { 1) Aprendizaje de accio nes interpersonales } \\
\text { (integra los dos aprendizajes). }\end{array}$ & 1) Catarsis. & 1) Universalidad \\
\hline 2) Autocomprensión de sí mismo & $\begin{array}{l}\text { 2) Autodescubrimiento } \\
\text { (Adicionada). }\end{array}$ & 2) Altruismo \\
\hline $\begin{array}{l}\text { 3) Cohesión grupal } \\
\text { 4) Aprendizaje Vicariante } \\
\text { (Sust. a Identificación). }\end{array}$ & & $\begin{array}{l}\text { 3) Instalación de esperanza. } \\
\text { 4) Guía. }\end{array}$ \\
\hline Reagrupación de McKenzie (1987). & & \\
\hline
\end{tabular}

Este autor explica que los grupos efectivos utilizan la categoría de Trabajo psicológico más que los no efectivos. No obstante según su renovada reagrupación de la primera categoría, en nuestro grupo de alco- hólicos existen la de Aprendizaje Interpersonal, la Comprensión de sí mismo, y también la Cohesión grupal, ello explicaría que el paciente alcohólico, se rehabilita porque estos procesos se han desarrollado en 
grupo. Ocurre otro tanto con la otra muestra en la que existen también la Comprensión de sí mismo, la Identificación (en McKenzie Aprendizaje vicariante), explicando el buen resultado de estos grupos en la práctica. Todo parece indicar que en nuestras muestras parece recogerse un trabajo respecto al factor Revalidac. de la Familia, el cual no aparece por ninguna parte en estos estudios, lo cual pudiera deberse a la importancia que para el ambiente latino tiene la necesidad de reconstrucción familiar, lo cual no parece existir en los estudios anglosajones citados en estas comparaciones.

1.6) Sin embargo en un estudio realizado en contexto español con el método de Yalom por MassHesse (1997), no se recoge entre las primeras cinco categorías la Revalidación familiar. En el mismo se ha encontrado lo siguiente y ha sido comparado con los encontrado en nuestro estudio:

Tabla VI: Comparación entre los resultados de FACTORES CURATIVOS obtenidos por Mass Messe en España y los resultados en Cuba.

\begin{tabular}{|lll|}
\hline Mas Hesse (1997): & Alcohólicos: & Psiquiátricos: \\
\hline 1) Universalidad & 1) Altruismo & 1) Altruismo \\
2) Aprendizaje interpersonal & 2) Comprensión de sí & 2) Comp. de sí mismo Aprend. Int. Ins. \\
3) Comprensión de sí mismo & 3) Revalidac. de la Familia. & 3) Revalidac. de la F. \\
4) Expresar sentimientos & 4) Aprend. Int. Ins & 4) Identificación. \\
5) Cohesión Grupal. & 5) Cohesión Grupal. & 5) Aprend. Int. Prod. \\
\hline
\end{tabular}

Obteniéndose que en nuestra muestra de alcohólicos existen tres categorías de FC que están presentes en este, mientras que en nuestra muestra de psiquiátricos existen solamente dos.

1.7) McKenzie (1987) ha expresado que los grupos ingresados sitúan el Altruismo e Infundir Esperanzas, como más altos en sus punteos, para ello toma como referencia el estudio de Maxmen (1979), sugiriendo un fuerte énfasis en los Factores Morales no Específicos como estos. Agrega este autor que el factor Insight es elevado su puntaje en psicoterapia de grupo ambulatoria a largo término en Bloch y Crouch, 1985; Ekert et al, 1981; Rohrbaugh y Bartels, 1975; Weiner, 1974, cit, en McKenzie, 1987. Sin embargo recoge McKenzie (1987) que en un interesante estudio de McKaskill en 1982, ocurrió un proceso totalmente diferente en un grupo de pacientes borderline, quienes escogieron las cinco categorías en el orden siguiente: 1) Insight; 2) Altruismo; 3) Esperanzas; 4) Catarsis y 5) Fact. Existenciales. Cita Mckenzie (1987), cinco estudios en forma de metanálisis con los resultados siguientes:

\section{Tabla VII: Metanálisis de los resultados de FACTORES CURATIVOS recogidos por varios autores en cinco estudios}

(Metanálisis de Cinco Estudios (Bloch y Crouch, 1985; Ekert, et al, 1981; Rohrbaugh y Bartels, 1975; Weiner, 1974; Yalom, 1985)
1) Catarsis.
2) Aprendizaje Interpersonal Ins.
3) Insight.
4) Aprendizaje Interpersonal Prod.

Nuestro estudio es similar en ambas muestras sólo en dos categorías de factores, el Aprendizaje Int. Ins está presente en los alcohólicos, mientras que en los psiquiátricos están presentes los dos tipos de Aprendizaje Int. Insumo y Producto. De lo cual podemos inferir que en nuestros grupos se dan las categorías de Aprendizaje Int. lo cual coincide en esto con los estudios de psicoterapia de grupo a largo término de la literatura. Ahora bien no estamos de acuerdo con McKenzie en que el Altruismo es propio de grupos ingresados, ya que en nuestras dos muestras es el primero en el puntaje.
Todo parece indicar que los Factores Curativos no parecen operar tal y como plantea este autor, sino que se da una combinación de varias categorías, en nuestro caso Trabajo psicológico con Morales no específicas.

1.8) Existen pocos estudios en la literatura sobre Factores Curativos en alcohólicos, entre ellos el de Feeney y Dranger (1976, cit. en Flores, 1997) con alcohólicos ingresados, y el de Kanas y Barr (1981, cit. en Flores, 1997) con alcohólicos ambulatorios, a continuación compararemos los encontrados con los nuestros: 
Tabla VIII: Comparación entre los FACTORES CURATIVOS recogidos por Feeney y Drangert y Kansas y Barr con los resultados cubanos.

\begin{tabular}{|lll|}
\hline (*) Feeney y Dranger (1976): & $\left(\mathbf{( *}^{*}\right)$ Kanas y Barr (1981): & Alcohólicos: \\
\hline 1) Catarsis & 1) Instalac. de Esperanza & 1) Altruismo \\
2) Insight & 2) Altruismo & 2) Comprensión de Sí- mismo \\
3) Ap. Int. Ins. & 3) Cohesión Grupal. & 3) Revalidación de la familia \\
4) Cohesión & 4) Aprendizaje. Int. Prod. & 4) Aprendizaje Int. Ins. \\
5) Inst. de Esperanza & 5) Universalidad & 5) Cohesión de grupo \\
\hline$\left(^{*}\right) ;(* *):$ Tomados de Flores (1997). & &
\end{tabular}

De la cual podemos inferir que existen grandes semejanzas entre los resultados del estudio de Kanas y Barr con alcohólicos ambulatorios y el nuestro, ya que están presentes o coinciden en tres Factores Curativos: Altruismo, Cohesión, y Aprendizaje interpersonal. El estudio de Feeney y Dranger coincide sólo en dos: Aprendizaje Interpersonal, y Cohesión, sin embargo, los tres estudios coinciden en estos dos últimos, de lo que podemos inferir que estos son extremadamente importantes para el alcohólico en su rehabilitación. Podemos inferir también que las semejanzas en resultados entre Kanas y el nuestro es debido a las similitudes entre variables como: 1) tipo de paciente, alcohólico; 2) tipo de terapia grupal: ambulatoria; 3) terapia interactiva de grupo.

2) En segundo lugar analizaremos los 10 ítems de más elevado puntaje en la muestra de alcohólicos, y haremos una interpretación de lo mismo.

2.1) De los 10 ítems 3 pertenecen a la Categoría de Yalom de Aprendizaje Int. (It 21; 22) ; A. Int. Producto y (It 17) a A. Int. Ins. ; tres pertenecen a la categoría Revalidación de la familia (It 42,44,45), lo cual está vinculado a la necesidad del paciente alcohólico de una reconstrucción de su familia, generalmente destruida, dividida, corroborando esto el hecho de que el FC Aprendizaje Int. Ins. punteó como el 4to más importante, y el FC de Revalidación Familiar como el 3ro más importante.

2.2) Es curioso el hecho de que el ítem 6 (FC Cohesión Grupal) y el 44 (Revalidación de la Familia) relativos al terapeuta, uno respecto al consejo del mismo, y el otro respecto a la admiración por el terapeuta, clasifican como el 8vo más importante, y el 9no más importante, de lo cual se infiere son demostraciones de transferencia positiva, la cual es necesaria siempre en grupo, y al parecer altamente importante para los pacientes alcohólicos rehabilitados.

2.3) La Cohesión Grupal está presente en el ítem 6 y en el 10, lo cual los clasificó en los lugares 8 vo y 10 mo respectivamente, de lo cual se infiere que la unión de los miembros es un gran factor también para rehabilitación del alcohólico, lo cual se refleja también en que este factor puntuó como el 5to más importante. d) Infundir esperanzas está presente en dos ítems de los 10 (it 51 y 55) en los lugares 2 do y 5to, de lo cual se infiere el alcohólico aspira a tener una vida mejor a través del tratamiento y la rehabilitación.

2.4) El ítem de más alta puntuación es el it 2, que pertenece al FC Altruismo, de lo cual inferimos que el paciente alcohólico recibe dando, y viceversa y esto es vital para su verdadera rehabilitación.

En cuanto a la variable tiempo, no se recogen diferencias relevantes entre las dos muestras, lo cual es posible se debe a lo pequeño de las muestras, por tanto estos resultados no son importantes a nuestros efectos.

Todo ello es relevante desde el punto de vista terapéutico para el terapeuta de grupo, ya que a partir de estos resultados es posible trazar estrategias de grupo para esta patología que estén encaminadas a:

1) El desarrollo del Altruismo ocurre por sí solo, pero también en la medida que otros pacientes hagan gala de este en el seno de las sesiones, y el terapeuta puede contribuir en gran medida a esto.

2) La comprensión de sí mismo, que expresa la necesidad del paciente de comprender la enfermedad que tiene y sus características, y el terapeuta debe hacer énfasis en esto con conferencias instructivas sobre el tema.

3) Hacer énfasis en el factor Aprendizaje interpersonal, o sea facilitar y desarrollar este con técnicas en grupo.

4) Un trabajo terapéutico más profundo con la familia, encaminado a la educación de la familia en el manejo del paciente y su enfermedad, muchas veces mal comprendida, lo cual obstaculiza la rehabilitación.

5) Expresa la necesidad de desarrollar la cohesión grupal para poder lograr el éxito en la rehabilitación, lo que se traduce en que el paciente debe aprender a pensar en grupo y para el grupo.

6) Un dato relevante para las estrategias de grupo es el conocer que hay factores que se desarrollan con el tiempo, en un inicio durante los primeros $3 / 6$ meses, y otros como la identificación llevan más tiempo.

7) Es preciso trasmitir y desarrollar la idea en el paciente, de que los grupos mantienen la abstinencia 
y el status de equilibrio en el paciente, con efectos más duraderos a más tiempo de grupo, lo que se desprende de la comparación entre ambas muestras de alcohólicos, y su análisis cualitativo. El paciente que comprenda que la terapia de grupo es la medicina que debe tomar toda su vida, es posible que garantice su rehabilitación total, por que mantiene frescos los Factores Curativos y por ende los cambios estables. De un análisis cualitativo de los 10 ítems más importantes para alcohólicos se desprenden las siguientes observaciones:

1) El ítem de más alta puntuación pertenece al Factor Curativo Altruismo, lo cual concuerda con que este fue el Factor Curativo de más alta puntuación, de lo que inferimos que el más importante.

2) El Factor Curativo Revalidac. de la Familia tiene 4 ítems entre los diez más importantes, de lo cual inferimos que esto concuerda en que es otro de los de más alta puntuación, y por tanto de los más importantes Factores Curativos para la rehabilitación del paciente alcohólico. Sin embargo debe señalarse que el ítem 6 y el 44, considerados por Yalom como parte del Factor Curativo Revalidac. de la Familia, hacen alusión al terapeuta, e implican una transferencia positiva presente hacia el terapeuta, y realmente discrepamos con Yalom en que este ítem deba pertenecer al Factor Curativo Revalidac. de la Familia, pensamos entonces que dada la importancia de este hallazgo, debiera crearse otra categoría de Factor Curativo que pudiera denominarse "Factor Transferencial ", pues la importancia de este es evidente y no existe dentro de los Factores Curativos. Sin embargo no aparece dentro de los 10 ítems más importantes de los pacientes psiquiátricos, de lo cual se desprende que no tiene la misma importancia para estos que tiene para los alcohólicos, entonces pensamos pudiera crearse una versión del instrumento de Yalom con cuatro ítems más, para alcohólicos en específico.

\section{CONCLUSIONES}

1) Nuestro trabajo demuestra que la terapia de grupo en alcohólicos a largo término ofrece resultados efectivos por la presencia de determinados Factores Curativos de grupo como: 1) Altruismo; 2) Comprensión de Sí-mismo; 3) Revalidación de la Familia; 4) Aprendizaje Int. ; 5) Cohesión grupal.

2) Se puede afirmar que el Factor Curativo Altruismo es la piedra angular en grupos de terapia para estos pacientes, ya que puntúa como el más alto, y es que este paciente recibe dando.

3) Se puede afirmar que el factor Revalidación de la Familia ocupa una posición básica entre las necesidades psicológicas del paciente alcohólico, por su posición entre las categorías y por estar contenido en tres ítems de los 10 más altos.

4) Se puede afirmar que existen Factores Curativos correspondientes a la categoría Trabajo psicológico que son básicos para la recuperación o rehabilitación de este paciente como Comprensión de sí mismo, y Aprendizaje Interpersonal.

5) Los resultados obtenidos por nosotros en la muestra control de pacientes psiquiátricos recoge una alta coincidencia con el trabajo original y antológico de Yalom, al coincidir en 3 Factores Curativos de los cinco más importantes: Altruismo, Aprendizaje Interpersonal Insumo, Aprendizaje Interpersonal Producto, lo cual nos demuestra que estos Factores Curativos son universales, ya que pueden producirse independientemente del medio cultural y social.

6) Los resultados obtenidos por nosotros en la muestra control de pacientes psiquiátricos coincide en 4 Factores Curativos: Altruismo, Comprensión de sí mismo, Revalidación de la familia, Aprendizaje Interpersonal Insumo, lo cual nos indica que estos Factores Curativos son en nuestro medio los más relevantes, y por tanto los que más deben ser trabajados en la psicoterapia grupal.

7) El Factor Curativo Revalidación Familiar constituye una de las preocupaciones más importantes para los pacientes alcohólicos y psiquiátricos en su tratamiento en nuestro medio, lo que es propio de nuestra cultura cubana y Latinoamericana, donde la familia es muy importante, ya que aparece como el tercero de más alta puntuación en ambas muestras, y cuatro veces entre los 10 ítems más relevantes, ya que no aparece entre los cinco primeros en ninguno de los estudios anglosajones de Norteamérica, ni tampoco en el único estudio hispano que registramos.

8) Se recoge que debe hacerse otra categoría de Factor Curativo para alcohólicos, que debe llamarse Factor Transferencial, ya que existe en alcohólicos una necesidad de transferencia positiva con el terapeuta para su rehabilitación.

9) Se confirma el método de evaluación del Cuestionario de Factores Curativos de Yalom como válido para el estudio de los procesos de grupo de la específicamente en alcohólicos.

10) Se recoge que las puntuaciones de los alcohólicos son más altas que las de los psiquiátricos ya que los alcohólicos perciben más intensamente los Factores Curativos que los psiquiátricos, porque sus necesidades de rehabilitarse son mayores.

11) Se recoge en contradicción con la literatura, que existe en los grupos ambulatorios una combinación de las categorías Trabajo psicológico, con Morales no específicas. 


\section{BIBLIOGRAFÍA}

Agazarian, I., Carter, F. B. (1993): Discussions on the large group. Group, Vol 17, N 4, pp. 210-234.

Alfano, A.; Thurstin, A. (1989): Program evaluation Research in ongoing Alcoholism Treatment: A Summary of Tuscalosa VA Project. Int. J. of the Addictions, 24 (4), pp. 303-314.

Avila Espada, A. (1993): La contribución del grupo en la Psicología Clínica y Comunitaria. (EL grupo psicoanalítico y sus modalidades técnicas en la Psicología Clínica y Comunitaria). Manual de Psicoterapia de grupo Analítico-Vincular. Madrid: Quipú Ed.

Bach, G. (1958): Psicoterapia Intensiva de Grupo. Ed Hormé, Buenos Aires.

Berne, E. (1985): Qué dice usted después de decir hola? Ed Grijalbo, Barcelona.

Bogani, E. (1984): Psicoterapia de grupo en el Alcoholismo. Drogalcohol, Vol. IX. Núm. 2.

Brown, S. (1985): Treating the Alcoholic. Ed. Wiley and Son, New York.

Brown, S. (1988): Treating Adult Child of Alcoholics. Ed. Wiley and Son, New York.

Carr, W. (1993): Some consequences of conceiving society as a large group. Group. Vol 17, N 4. pp. 235-244.

Colli, M., Zaldívar, D. (2002): Revisión crítica de los efectos de la psicoterapia de grupo en la literatura: sus variables, instrumentos, y criterios de investi gación. Rev. Cubana de Psicología (en prensa)

Cuadrado, P. (1998): Evolución de la dependencia alcohólica en tratamiento. Factores predictivos en un seguimiento de 5 a 7 años. Adicciones, Vol. 10, Num. 4, pp. 335-341.

Flores, Ph. (1997): Group Psychotherapy with Addicted Populations. Ed. Ha worth Press, New York.

González Menéndez, R. (1997): S. O. S. Toxicómanos. Ed. Científico-Técnica, La Habana.

González Menéndez, R. ;Goicolea, S. (1995): El dependiente alcohólico primario cinco años después de su primer ingreso. Rev. Esp. de Drogodependencia 20 (2), pp. 133-140.

Golden, S. ; Halliday, K. ; Khantzian, E. et all. (1995): Terapia de grupo dinámica para pacientes adictos a sustancias: Reconceptuación. En Psicoterapia de Grupo en la práctica clínica, Alonso, A. y Zwiller, H. Comp. Ed Manual Moderno. México, D. F.

Hernández Boado, M. (1989): Estructuración de un centro de Asistencia para la Rehabilitación de enfermos y enfermas alcohólicas. En Rev. Esp. Drogodependencias, 14 (4), pp. 249-255.

Joyce, A. ;Azim, H. ;Morin, H. (1988): Brief Crisis Group Psychotherapy Versus the initial Sessions of Long-Term Group Psychotherapy: An Exploratory compa rison. Group, Vol 12, Number 1, Spring. pp. 1-19.

Kahn, M. (1986): Inpatient group psychotherapy: which type of group is best?. En Group, Vol 10, N 1, Spring pp. 2733.

Klein, E. (1993): Large groups in treatment and training sittings. Group, Vol 17, N 4, pp. 198-209.
Khantzian, E. (1989): From theory to practice: The planned Treatment of drug users. Interview by Dr. Craigh Burrell. Int. Journal of Addictions, 24 (4), 351-383.

Lawrence, G. (1993): Signals of trascendence in large groups. Group, Vol 17, N 4, pp. 254-266.

Lescz, M. (1987): Interactional group Psychotherapy with non psychotic inpatients. Group, Vol 10, No 1, Spring, pp. 13-20.

Martín, A. (1996): Anexo 1. Criterios para diagnóstico del Alcoholismo CIE-10. Síndrome de dependencia por el alcohol (F10. 2). En Programa de actualización en Medicina de Familia y Comunitaria. Ed. Doyma, Barcelona.

Marlatt, G., Gordon, J. (1985): Relapse Prevention. Ed. Guilford Press, Washing ton.

Martínez, A. y Fernández, J. M. (1996): Grupos de Psicoterapia extrahospi talaria. En 30 Años de Alcohol y otras Drogas en Asturias. Ed. Por Bobes, J. et all. Oviedo.

MásHesse, J. (1997): Integración de psicoterapias en un hospital de día. Valoración de tratamientos. En “Psicoterapias en el sector público: un marco para su integración". Asociación Española de Neuropsiquiatría, Estudios 19. A. Fernández Liria y cls. comp.

Mathiesen, E., Davenport, Y. (1988): Reciprocal Depression in recovering alcoholic couples: The Efficacy of Psychodinamic Group Treatment. Group, Vol 12, N 1. Spring, 45-54.

McKenzie, K. R. (1987): Therapeutic factors in group psychotherapy: a con temporary view. Group, Vol 11, No 1, Spring, pp. 25-34.

McKenzie, K. R. (1990): Time -limited Group Psychotherapy. Ed. American Psychiatric Press Inc., Washington.

McKenzie, K. R. ;Tschuschke, V. (1993): Relatedness, Group work, and Outcome in Long-Term Inpatient Psychotherapy Groups. Journal of Psychotherapy Practice and Research, Vol 2, N 2, Spring.

Monrás, M. ; Freixa, N. ; et all. (2000): Eficacia de la terapia de grupo para alcohólicos. Resultados de un ensayo clínico controlado. Medicina Clínica, Vol 115, N 4, pp. 126-131.

Mullan, H. (1963). Group Psychotherapy with the alcoholic. Current Psychiatric Therapies. Vol III . Masserman, J. Ed.

Pattison, M. (1965): Evaluation Studies of Group psychotherapy. Int. J. of Group Psychotherapy, Vol XV, N. 3, July, . pp. 382-397.

Pellegrini, J. L. (1997): Alcoholismo y G. I. A.

Potts, F. (1960): Psicoterapia de grupo e Introducción a la Sociometría. Imprenta Nacional de Cuba.

Rodríguez Mesa, R. (1982): Psicoterapia de Grupo. Experiencia con grupos numerosos. Revista del Hospital Psiquiátrico de La Habana. Vol. XXIII, No 1, EneroMarzo.

Rodríguez Mesa, R. (1988): Psicoterapia de grandes grupos. En Psicoterapia. Ed ECIMED, La Habana.

Rodríguez Mesa, R. (1997): Del Psicoanálisis a la Psicoterapia de Grupo. Tesis Doctoral, Biblioteca Médica, MINSAP, Cuba (no publicada). 
Sierra, E. (1978): Estudio observacional sobre grupos de discusión en enfermos alcohólicos. Drogalcohol, Vol. III, Núm 4.

Smith, F. ; McNiel, D. ;Binder, R. (1988): Therapeutic Factors in Two Forms of Inpatient Group Psychotherapy: Music Therapy and Verbal Therapy. Group, Vol 12, N. 3, Fall, pp. 145-156.

Steiner, C. (1970): Games alcoholics plays. Ed. Ballantine Books.

Steiner, C. (1992): Los guiones que vivimos. Ed. Kairós, Barcelona.

Tschuschke, V. ; Dies, R. (1994): Intensive analysis of Therapeutic Factors and Outcome in Long-Term Inpatient Groups. Int. Journal of Group Psycho therapy, 44 (2).

Tschuschke, V. ; Dies, R. (1997): The contribution of Feedback to Outcome in Long-Term Group Psychotherapy. Group, Vol 21, N 1.
Vannicelli, M. (1995): Group Psychotherapy with Substance Abusers and Family members. En Psychotherapy and Substance Abuse, A practitioners' s Handbook. Ed A. Washton, pp. 337-335.

Vannicelli, M. (1982): Group Psychotherapy with alcoholics: special techniques. Journal of Studies on Alcohol, 40, 457-471.

Vannicelli, M. (1987): Treatment of alcoholic couples in outpatient Group Therapy. Group, Vol 11, Num 4, Winter, pp. 247-257.

Vinogradov, S. and Yalom, I. D. (1990): Concise guide of Group Psycho therapy. Ed. American Psychiatric Press Inc., Washington.

Yalom, I. D. (1986): Teoría y Práctica de la Psicoterapia de Grupo. Fondo Cultura Económica. México.

Zaldívar, D. (1998): La Terapia Grupal Combinada como alternativa en el trata miento del alcohólico. Revista Cubana de Psicología, Vol 15, No. 1., 29-37. 
